\title{
TORSIONAL VIBRATIONS OF NONHOMOGENEOUS MAGNETOSTRICTIVE ELASTIC CIRCULAR CYLINDER
}

\author{
A.N. ABD-ALLA \\ Department of Mathematics \\ Faculty of Science at Sohag \\ Sohag, Egypt \\ and \\ LOKENATH DEBNATH \\ Department of Mathematics \\ University of Central Florida \\ Orlando, Florida 23816, U.S.A. \\ (Received July 7, 1992 and in revised form September 1, 1993)
}

\begin{abstract}
This paper is concerned with the torsional vibrations of a nonhomogeneous magnetostrictive elastic cylinder. The cylinder is subjected to the action of a circumferential magnetic field produced by an axial current of constant density and the deformation of a magnetostrictive cylinder is produced by a constant longitudinal magnetic field. The frequency equation is determined, and the displacement and stress components are numerically calculated with graphical presentations.
\end{abstract}

KEY WORDS AND PHRASES: Torsional vibration, magnetostrictive cylinder 1991 AMS SUBJECT CLASSIFICATION CODES: 73D35

\section{INTRODUCTION}

The study of torsional deformation of magnetostrictive cylinder has received some attention in recent years. Recently, devices such as the "Twistor" in the field of digital computer use the magnetostrictive effect as shown by Lewis [1]. Motivated by this work, a study is made of torsional vibrations of a nonhomogeneous magnetostrictive elastic cylinder. This study is based on simplified version of nonlinear magnetoelastic equations and the constitutive relations considered by Abd-Alla and Maugin [2] and Maugin [3]. Further, the cylinder is subjected to the action of a circumferential magnetic field produced by an axial current of constant density and the deformation of a magnetostrictive cylinder is produced by a constant longitudinal magnetic field. The associated frequency equation is determined. The displacement and stress components are numerically calculated with graphical presentations.

\section{BASIC EQUATIONS}

Making reference to [2], we denote the density of magnetostrictive elastic solid by $\rho$, the magnetic induction by $\mathcal{B}_{K}$ the Poila-Kirchhoff stress tensor by $T_{K L}$, the tensor of elasticity coefficients by $C_{K L M N}$, the tensor of coefficients of magnetostriction by $B_{K L M N}$, and the tensor of magnetic susceptibilities by $\chi_{K L}$. For the usual magnetostriction, we consider the linear terms in $\nabla \mathbf{u}$ and the quadratic terms in the magnetic field $\mathbf{H}$. The equations of motion and equations of magnetic field are given by Abd-Alla and Maugin [2] in the form

$$
\begin{gathered}
\rho \frac{\partial^{2} u_{L}}{\partial t^{2}}=T_{K L, K}, \\
\mathcal{B}_{K \cdot K}=0,
\end{gathered}
$$


where commas are used to denote differentiation with respect to the Cartesian coordinates. The constitutive relations are

$$
\begin{gathered}
T_{K L}-C_{K L M N} u_{M, N}+\mathcal{B}_{K L M N} H_{M} H_{N}, \\
\mathcal{B}_{K}=\mu_{K L} H_{L}-\mathcal{B}_{K L M N}^{0} H_{L} u_{M, N},
\end{gathered}
$$

where

$$
\begin{aligned}
B_{K L M N} & =\frac{1}{2} B_{K L M N}+\chi_{L N} \chi_{K M}+\left(\delta_{K M} \delta_{N) L}-\frac{1}{2} \delta_{N M} \delta_{K L}\right), \\
B_{K L M N}^{0} & =B_{K L M N}+\delta_{L M} \delta_{N K}+\delta_{K M} \delta_{N L}+\delta_{K L} \delta_{N M}, \\
\mu_{K L} & =\delta_{K L}+\chi_{K L} .
\end{aligned}
$$

It follows from result (2.5a) that due to the quadratic nature of magnetostriction, the magnetic contribution to $(2.5 a b c)$ comes from three effects: (i) magnetostriction, (ii) the fact that the body is magnetized $\left(\chi_{K L} \neq 0\right)$, and (iii) the Lorentz force that yields the contribution represented by the term within the parentheses in (2.5a). We use the simplified version of the anisotropic nonlinear magnetoelastic equations (2.1)-(2.2) and the constitutive relations (2.3)-(2.5abc) and obtain the simplest stress-displacement-magnetostrictive field relations which are valid for an isotropic nonhomogeneous and magnetostrictive medium in the form

$$
T_{K L}=\lambda \delta_{K L} u_{M, M}+2 \mu u_{K, L}+2 p H_{K} H_{L}-\frac{1}{2} \delta_{K L} H_{M} H_{M},
$$

where

$$
\begin{gathered}
C_{K L M N}=\lambda \delta_{K L} \delta_{M N}+\mu\left(\delta_{K N} \delta_{L M}+\delta_{K M} \delta_{L N}\right), \quad B_{K L M N}=b_{0} \delta_{K M} \delta_{L N}, \mu_{K L}=\chi_{0} \delta_{K L}, \\
p=\frac{1}{2}\left(\frac{1}{2} b_{0}+\chi_{0}^{2}+1\right)
\end{gathered}
$$

In terms of the cylindrical polar coordinates $(r, \theta, z)$, the following constitutive relations describe the interaction of the elastic and magnetic fields so that the stress components are

$$
\begin{aligned}
& T_{r r}=\lambda \varepsilon+2 \mu \frac{\partial u_{r}}{\partial r}+2 p H_{r}^{2}-\frac{1}{2} \alpha \\
& T_{\theta \theta}=\lambda \varepsilon+2 \mu\left(\frac{u_{r}}{r}+\frac{1}{r} \frac{\partial u_{\theta}}{\partial \theta}\right)+2 p H_{\theta}^{2}-\frac{1}{2} \alpha \\
& T_{z z}=\lambda \varepsilon+2 \mu \frac{\partial u_{z}}{\partial z}+2 p H_{z}^{2}-\frac{1}{2} \alpha \\
& T_{r \theta}=\mu\left(\frac{1}{r} \frac{\partial u_{r}}{\partial \theta}+\frac{\partial u_{\theta}}{\partial r}-\frac{u_{\theta}}{r}\right)+2 p H_{r} H_{\theta} \\
& T_{r z}=\mu\left(\frac{\partial u_{z}}{\partial r}+\frac{\partial u_{r}}{\partial z}\right)+2 p H_{r} H_{z} \\
& T_{\theta z}=\mu\left(\frac{\partial u_{\theta}}{\partial z}+\frac{1}{r} \frac{\partial u_{z}}{\partial \theta}\right)+2 p H_{\theta} H_{z}
\end{aligned}
$$

where

$$
\varepsilon=\frac{\partial u_{r}}{\partial r}+\frac{u_{r}}{r}+\frac{1}{r} \frac{\partial u_{\theta}}{\partial \theta}+\frac{\partial u_{z}}{\partial z}, \quad \alpha=H_{r}^{2}+H_{\theta}^{2}+H_{z}^{2},
$$

where $\mathbf{u}=\left(u_{r}, u_{\theta}, u_{z}\right)$ is the displacement field victor, $\lambda, \mu$ are elastic constants and $H$ 's are the components of magnetic field. 
The equations of motion in cylindrical polar coordinates are

$$
\begin{gathered}
\frac{\partial T_{r r}}{\partial r}+\frac{1}{r} \frac{\partial T_{r \theta}}{\partial \theta}+\frac{\partial T_{r z}}{\partial z}+\frac{T_{r r}-T_{\theta \theta}}{r}=\rho \frac{\partial^{2} u_{r}}{\partial t^{2}}, \\
\frac{\partial T_{r \theta}}{\partial r}+\frac{1}{r} \frac{\partial T_{\theta \theta}}{\partial \theta}+\frac{\partial T_{\theta z}}{\partial z}+\frac{2}{r} T_{r \theta}=\rho \frac{\partial^{2} u_{\theta}}{\partial t^{2}}, \\
\frac{\partial T_{r z}}{\partial r}+\frac{1}{r} \frac{\partial T_{\theta z}}{\partial \theta}+\frac{\partial T_{z z}}{\partial z}+\frac{1}{r} T_{r z}=\rho \frac{\partial^{2} u_{z}}{\partial t^{2}},
\end{gathered}
$$

\section{TORSIONAL VIBRATIONS}

We take the positive direction of the $z$-axis along the axis of the cylinder, and assume that the displacement vector $u$ has its only non-vanishing component $u_{\theta}$ which is independent of $\theta$. This means that the normal cross sections ( $z=$ constant) of the cylinder are rotating with respect to each other without distortion. With the magnetostrictive cylinder of inner radius $r=b$, outer radius $r=a$ and of length $\boldsymbol{b}$, the boundary conditions are prescribed as

(i) The shearing force has the form

$$
\left(T_{\theta_{z}}\right)_{z-0, r-0}=f(r)= \begin{cases}0, & 0 \leq r \leq b \\ \beta r, & b \leq r \leq a\end{cases}
$$

where $\beta$ is a constant.

(ii) The mixed boundary conditions are in the following form

$$
u_{\theta}=0 \text { at } z-l, T_{r \theta}-0 \text { at } r-a \text {. }
$$

The second condition means that the curved surface $(r-a)$ of the cylinder is free from mechanical tractions.

(iii) The cylinder is subjected to the action of a circumferential magnetic field produced by an axial current of constant density $I_{0}$ and the deformation of a magnetostrictive cylinder is produced by a constant longitudinal magnetic field $H_{0}$. These can be expressed as

$$
H_{r}=0, H_{\theta}=\frac{1}{2} I_{0} r, H_{z}-H_{0} \text {. }
$$

(iv) In view of the non-homogenity, the rigidity $\mu$ and density $\rho$ are chosen in the following form

$$
\mu=\mu_{0} e^{-k x}, \rho=\rho_{0} e^{-k x} \text {, }
$$

where $\mu_{0}, \rho_{0}$ and $k$ are constants.

The only nonvanishing equation of motion is

$$
\frac{\partial^{2} u_{\theta}}{\partial r^{2}}+\frac{1}{r} \frac{\partial u_{\theta}}{\partial r}-\frac{u_{\theta}}{r^{2}}+\frac{\partial^{2} u_{\theta}}{\partial z^{2}}+\frac{\partial u_{\theta}}{\partial z}=\frac{1}{c^{2}} \frac{\partial^{2} u_{\theta}}{\partial t^{2}},
$$

where $c^{2}=\frac{\mu_{0}}{\mathrm{p}_{0}}$.

We seek separable solution for the displacement field in the form

$$
u_{\theta}=R(r) Z(z) e^{i \omega t} \text {. }
$$

Substituting (3.6) into (3.5) gives

$$
\frac{1}{R}\left(\frac{d^{2} R}{d} r^{2}+\frac{1}{r} \frac{d R}{d r}-\frac{R}{r^{2}}+\frac{\omega^{2}}{c^{2}}\right)=-\frac{1}{Z}\left(\frac{d^{2} Z}{d z^{2}}-k \frac{d Z}{d z}\right)=-m^{2},
$$

where $m$ is a separation constant. Equation (3.7) splits up into two equations, namely: 


$$
\begin{gathered}
\frac{d^{2} R}{d r^{2}}+\frac{1}{r} \frac{d R}{d r}+\left(q^{2}-\frac{1}{r^{2}}\right) R=0, \\
\frac{d^{2} Z}{d z^{2}}-k \frac{d Z}{d z}-m^{2} Z=0,
\end{gathered}
$$

where

$$
q^{2}=\frac{\omega^{2}}{c^{2}}+m^{2}
$$

The solutions for $R$ and $Z$ are

$$
R=A_{1} J_{1}(q r)+B_{1} Y_{1}(q r), \quad Z=C_{1} e^{\lambda_{1} z}+D_{1} e^{\lambda_{2} z},
$$

where

$$
\lambda_{1}, \lambda_{2}=\frac{1}{2}\left(k \mp \sqrt{k^{2}-4 m^{2}}\right),
$$

$A_{1}, B_{1}, C_{1}$ and $D_{1}$ are arbitrary constants and $J_{1}(x)$ and $Y_{1}(x)$ are Bessel functions of the first and second kind of order one, respectively.

Now, for a solid cylinder, $\frac{u_{0}}{r}$ must be finite at $r=0$, hence $B_{1}=0$, and therefore the solution of equation (3.5) can be written as

$$
u_{\theta}=J_{1}(q r)\left[C e^{\lambda_{1} z}+D e^{\lambda_{2} z}\right] e^{i \omega t}
$$

and the stress components are

$$
\begin{aligned}
& T_{r \theta}=\mu_{0} e^{-k z}\left[C e^{\lambda_{1} z}+D e^{\lambda_{2} z}\right] q J_{2}(q r) e^{i \omega t} \\
& T_{\theta z}=\mu_{0} e^{-k z}\left[\lambda_{1} C e^{\lambda_{1} z}+\lambda_{2} D e^{\lambda_{z} z}\right] J_{1}(q r) e^{i \omega t}+p J_{0} H_{0} r,
\end{aligned}
$$

where $C=A_{1} C_{1}$ and $D=A_{1} D_{1}$. It is noted that the influence of magnetic field is evident in the shearing stress component $T_{\mathrm{ez}}$ which is a function of the radial distance. Making use of the boundary condition (3.2b) we obtain the frequency equation

$$
J_{2}(q a)=0 .
$$

This equation has infinitely many roots, the first non-zero eight positive roots are given in Table 1 where $\varepsilon_{n}=(q a)_{n}, n=1,2, \ldots, 8$.

\begin{tabular}{|c|c|c|c|c|c|c|c|c|}
\hline$n$ & 1 & 2 & 3 & 4 & 5 & 6 & 7 & 8 \\
\hline$\varepsilon_{n}$ & 5.1362 & 8.4172 & 11.6198 & 14.7959 & 21.1170 & 24.4205 & 27.4205 & 30.5692 \\
\hline
\end{tabular}

Table 1.

Furthermore, the boundary condition (3.2a) gives

$$
C e^{\lambda_{1} l}+D e^{\lambda t}=0
$$

By virtue of condition (3.1ab), the following result follows from equation (3.15)

$$
\mu_{0}\left[C \lambda_{1}+D \lambda_{2}\right] J_{1}(q r)+p J_{0} H_{0} r=f(r) \text {. }
$$

Multiplying both sides of (3.18) by $r J_{1}(q r)$ and then integrating in the range $(0, a)$ we get

$$
C \lambda_{1}+D \lambda_{2}=\frac{1}{\mu_{0} \gamma} \int_{0}^{a} r f(r) \nu_{1}(q r) d r
$$


where we have used the following results [4]:

$$
\gamma=\int_{0}^{a} r J_{1}^{2}(q r) d r=\frac{a^{2}}{2}\left\{J_{1}(q a)\right\}^{2}, \quad \int_{0}^{a} r^{2} J_{1}(q r) d r=0 .
$$

Now, using the condition (3.1), equation (3.19) becomes

$$
C \lambda_{1}+D \lambda_{2}--\frac{\beta b^{2}}{\mu_{0} \gamma} J_{2}(q b) \text {. }
$$

The constants $C, D$ can be determined by solving the two equations (3.17) and (3.21) so that

$$
C=\frac{\Delta_{C}}{\Delta}, \quad D=\frac{\Delta_{D}}{\Delta},
$$

where

$$
\Delta_{C}=\frac{\beta b^{2}}{\mu_{0} \gamma} J_{2}(q b) e^{\lambda_{1} l}, \quad \Delta_{D}=-\frac{\beta b^{2}}{\mu_{0} \gamma} J_{2}(q b) e^{\lambda_{1} l} \text { and } \Delta-\lambda_{2} e^{\lambda_{1} l}-\lambda_{1} e^{\lambda_{t} l} \text {. }
$$

\section{NUMERICAL RESULTS AND DISCUSSION}

The displacement and stress for the first mode of vibration are given below graphically. Computational work is carried out with the following data:

$$
\begin{array}{lll}
H_{0}=100 \mathrm{Am} / \mathrm{m} & , & I_{0}=100 \mathrm{Am} / \mathrm{m}^{2} \\
\mu_{0}=0.621 \mathrm{~N} / \mathrm{m}^{2} & , & \rho_{0}=3.22 \mathrm{Kg} / \mathrm{m}^{3} \\
k=0.05 & , & \beta=10^{5} \\
s / k=1 & , & k b=1 / 2 \\
l / a=2 & , & z / a=2 \\
q / a=5.13562 & , & p=0.2 \times 10^{-1}
\end{array}
$$

The displacement, $-u_{\theta} \times 10^{6}$ at different radii is shown in Fig. 1 . The stress components, $-T_{r \theta} \times 10^{5}$ and $-T_{\theta_{2}} \times 10^{4}$ are shown in Fig. 2 and Fig. 3 respectively, for fixed value of time $t=1$ sec. It is obvious from Fig. 1 that the maximum value of the displacement occurs near $r / a=0.36$ and the displacement changes its sign at the point $r / a=0.74$. The maximum values of $-T_{r \theta}$, from Fig. 2 , occurs near $r / a=0.6$. Moreover, from Fig. 3 , it follows that the component of stress $-T_{\theta z}$ has the maximum value near $r / a=0.35$ and it changes its sign at the point $r / a=0.65$ when there exists magnetostriction effect, and at the point $r / a=0.75$ when there is no magnetostriction. This means that the effect of the magnetostriction reduces the value of $-T_{0 z}$ and it becomes more sensitive with the increasing of the distance.

\section{REFERENCES}

[1] LEWIS, J. A. The small-field theory of the Joule and Wiedemann effects, Quart. Appl. Math. 1(1962), 13-20.

[2] ABD-ALLA, A. N. and MAUGIN, G. A. Nonlinear magnetoacoustic equations, J.Acoust. Soc. Amer. 82 (5) (1987), 1746-1752.

[3] MAUGIN, G. A. Continuum Mechanics of Electromagnetic Solids, North-Holland, Amsterdam (1988).

[4] WATSON, G. N. A Treatise on the Theory of Bessel Function 4th Ed., Cambridge University Press, London, 1966.

[5] BELTZER, A. I. Acoustics of Solids, Springer-Verlag, Berlin (1988). 


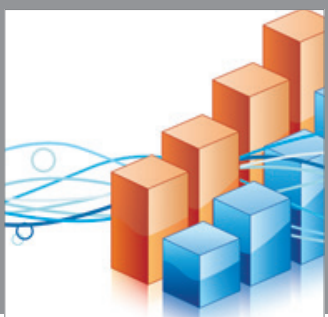

Advances in

Operations Research

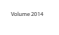

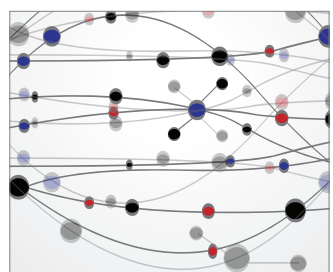

\section{The Scientific} World Journal
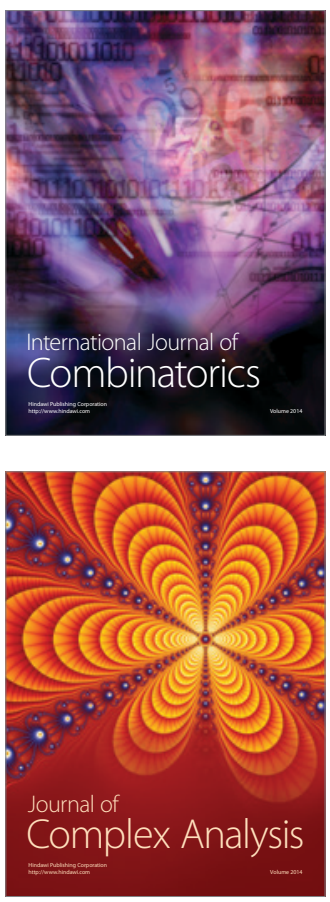

International Journal of

Mathematics and

Mathematical

Sciences
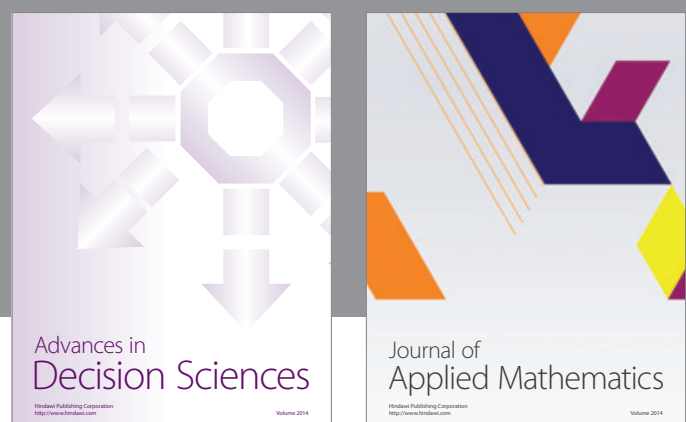

Journal of

Applied Mathematics
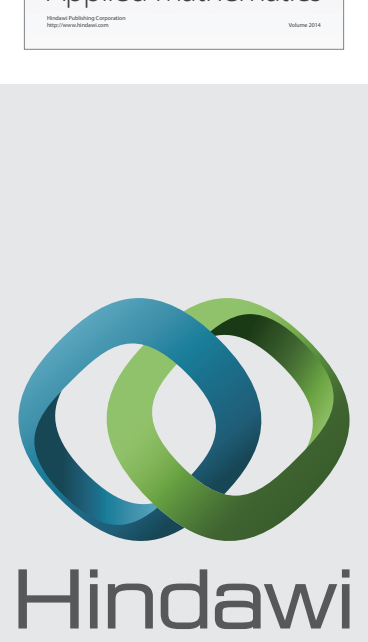

Submit your manuscripts at http://www.hindawi.com
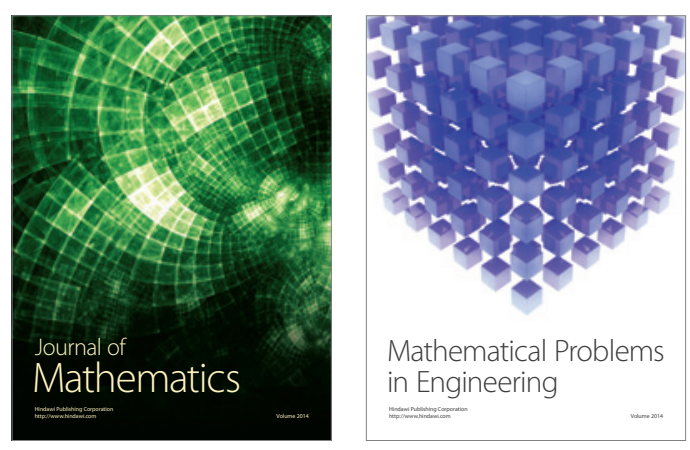

Mathematical Problems in Engineering
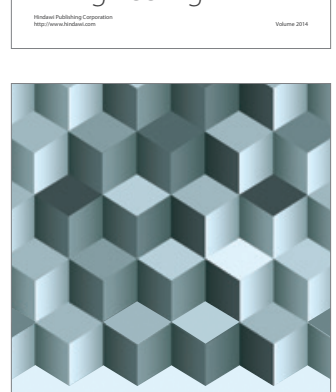

Journal of

Function Spaces
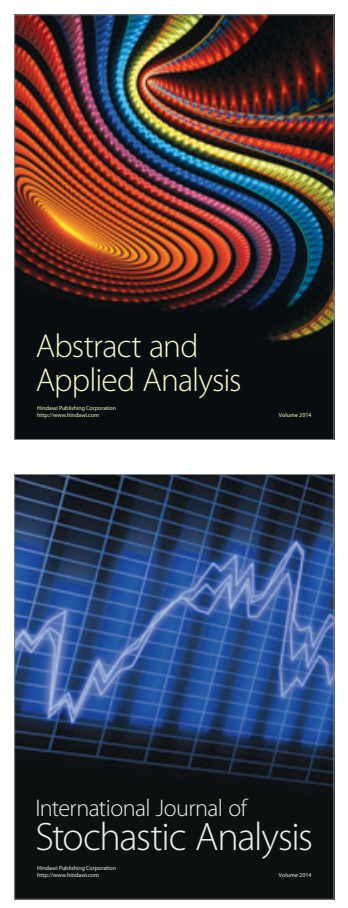

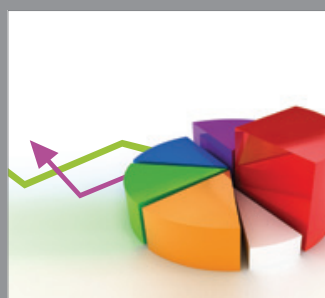

ournal of

Probability and Statistics

Promensencen
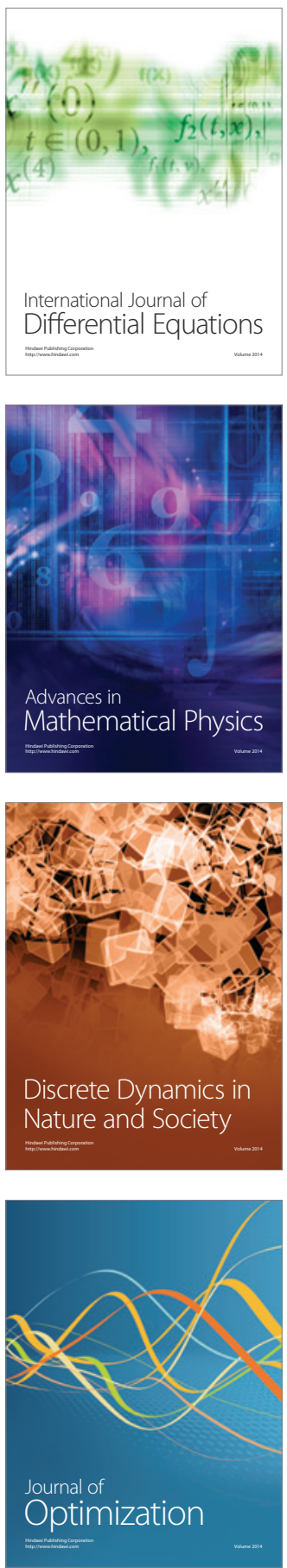